\begin{tabular}{|c|c|c|c|c|c|c|}
\hline \multirow{4}{*}{ Impact Factor: } & ISRA (India) & $=4.971$ & SIS (USA) & $=0.912$ & ICV (Poland) & $=6.630$ \\
\hline & ISI (Dubai, UAE & $=\mathbf{0 . 8 2 9}$ & РИНЦ (Russia) & $=0.126$ & PIF (India) & $=1.940$ \\
\hline & GIF (Australia) & $=0.564$ & ESJI (KZ) & $=8.716$ & IBI (India) & $=4.260$ \\
\hline & JIF & $=1.500$ & SJIF (Morocco) & $=5.667$ & OAJI (USA) & $=0.350$ \\
\hline
\end{tabular}

\section{SOI: 1.1/TAS DOI: $10.15863 /$ TAS \\ International Scientific Journal Theoretical \& Applied Science}

p-ISSN: 2308-4944 (print) e-ISSN: 2409-0085 (online)

Year: $2020 \quad$ Issue: 03 Volume: 83

Published: $30.03 .2020 \quad$ http://T-Science.org
QR - Issue

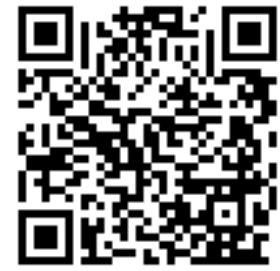

Makhmudzhon Melibaev

Namangan Engineering Construction Institute associate Professor, candidate of technical Sciences

Anvarzhon Dadakhozhaev

Namangan Engineering Construction Institute Associate Professor, candidate of agricultural Sciences

Ma"ruf Makhmudzhanovich Mamadzhonov Namangan Engineering Construction Institute senior lecturer

Sherzod Ergashalievich Khaydarov Namangan Engineering Construction Institute Teacher, city of Namangan, Uzbekistan inventor_uz@mail.ru

\title{
EXPERIMENTAL METHODS FOR DETERMINING DEFORMATIONS AND STRESSES OF TRACTOR WHEEL TIRES
}

Abstract: The deformation properties of pneumatic tire wheels are the main performance indicators. For experimental determination of deformations and stress, various methods are used. The most widely used are mechanical methods, which are based on measuring the movements of body points using tensometers: mechanical, electrical resistance, inductive, capacitive, etc [1,2].

Key words: deformation, pneumatic, dynamics, wheels, vertical load, cross-section, torque, surface, soil, load.

Language: Russian

Citation: Melibaev, M., Dadakhozhaev, A., Mamadzhonov, M. M., \& Khaydarov, S. E. (2020). Experimental methods for determining deformations and stresses of tractor wheel tires. ISJ Theoretical \& Applied Science, 03 (83), 138-144.

\section{Soi: http://s-o-i.org/1.1/TAS-03-83-30 Doi: crossef https://dx.doi.org/10.15863/TAS.2020.03.83.30 \\ Scopus ASCC: 2200. \\ ЭКСПЕРИМЕНТАЛЬНЫЕ МЕТОДЫ ОПРЕДЕЛЕНИЯ ДЕФОРМАЦИЙ И НАПРЯЖЕНИЙ ТРАКТОРНЫХ КОЛЁСНЫХ ШИН}

Аннотация: Деформационные свойства пневматических колёсных шин являются основными эксплуатационными показателями. Для экспериментального определения деформаций и напряжении используют различные способы. Наибольшее распространение получили механические методы, в основе которых лежит измерение перемещзений точек тела $c$ помощью тензометров: механических, электросопротивления, индуктивных, ёмкостных и др[1,2].

Ключевые слова: деформачии, пневматических, динамике, колеса, вертикальной нагрузки, сечения, крутящчих, поверхность, почв, нагрузка.

Введение

УДК 631.32.
Измерение временных деформаций и перемещений в большинстве случаев производится с помощью механических приборов 


\begin{tabular}{|c|c|c|c|c|c|c|}
\hline \multirow{4}{*}{ Impact Factor: } & ISRA (India) & $=4.971$ & SIS (USA) & $=0.912$ & ICV (Poland) & $=6.630$ \\
\hline & ISI (Dubai, UAE & $=0.829$ & РИНЦ (Russia) & $=0.126$ & PIF (India) & $=1.940$ \\
\hline & GIF (Australia) & $=0.564$ & ESJI (KZ) & $=8.716$ & IBI (India) & $=4.260$ \\
\hline & JIF & $=1.500$ & SJIF (Morocco) & $=5.667$ & OAJI (USA) & $=0.350$ \\
\hline
\end{tabular}

(индикатор часового типа), устанавливаемых нередко в зоне действия высокой нагрузки.

Для замера деформации шин в динамике нами разработана и применена установка (рис.1). Устройство и принцип работы прибора заключаются в следующем.

В систему входит рама, тарелки из металлического материала, индикаторы часовой модели, ограничитель с опусканием трактора и механизм для направления колеса трактора [3].

Для выполнения своих функций колесо трактора должно взаимодействовать с опорной поверхностью через зону контакта. Основная нагрузка, которую воспринимает шина, - это нормальная нагрузка. При малых и больших нагрузках в материалах шины увеличивается сопротивление качения [4,5].

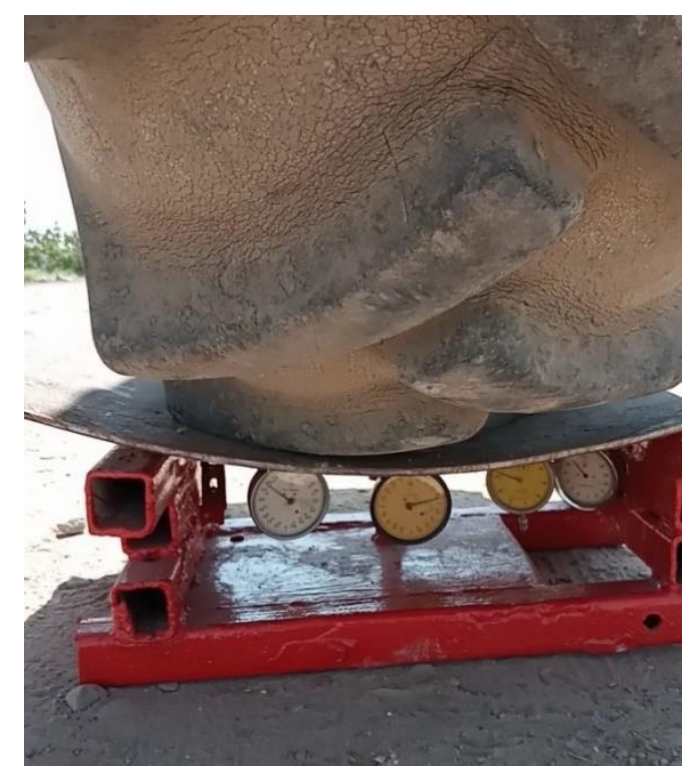

Рис.1. Индикатор часовой модели ИЧ-150 (давления в шинах 0,10 МПа) ведущего колеса 13,6 R 38 ЯР318 для определения деформационных свойств.

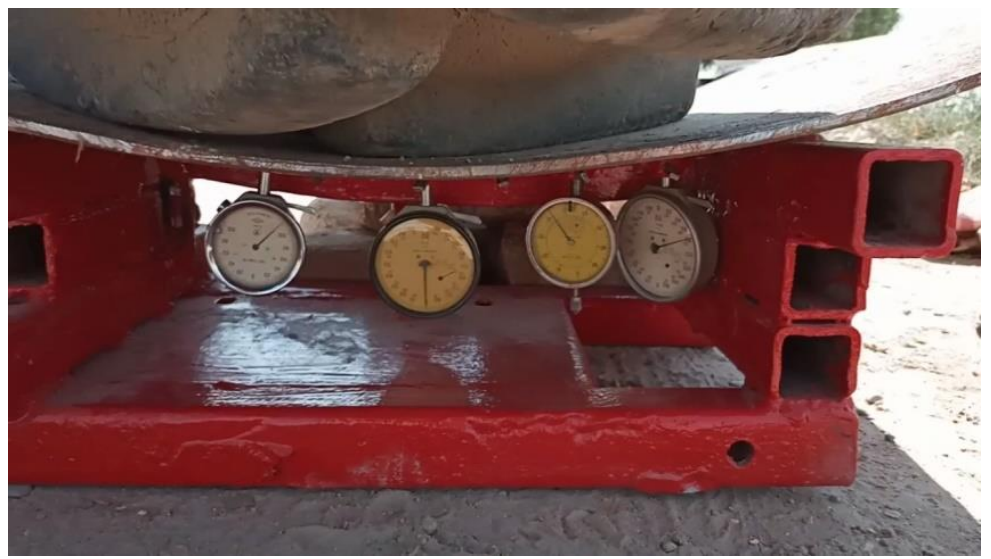

Рис.2. Ведущее колеса 18,4-34 TR-135 10PR New Holland TD5 110 для определения деформационных свойств при помощи индикатора часовой модели ИЧ-150 (давление в шинах 0,10 МПа) 


\begin{tabular}{|c|c|c|c|c|c|c|}
\hline \multirow{4}{*}{ Impact Factor: } & ISRA (India) & $=4.971$ & SIS (USA) & $=0.912$ & ICV (Poland) & $=6.630$ \\
\hline & ISI (Dubai, UAE & $=\mathbf{0 . 8 2 9}$ & РИНЦ (Russia & $=0.126$ & PIF (India) & $=1.940$ \\
\hline & GIF (Australia) & $=0.564$ & ESJI (KZ) & $=8.716$ & IBI (India) & $=4.260$ \\
\hline & JIF & $=1.500$ & SJIF (Morocco & $=5.667$ & OAJI (USA) & $=0.350$ \\
\hline
\end{tabular}
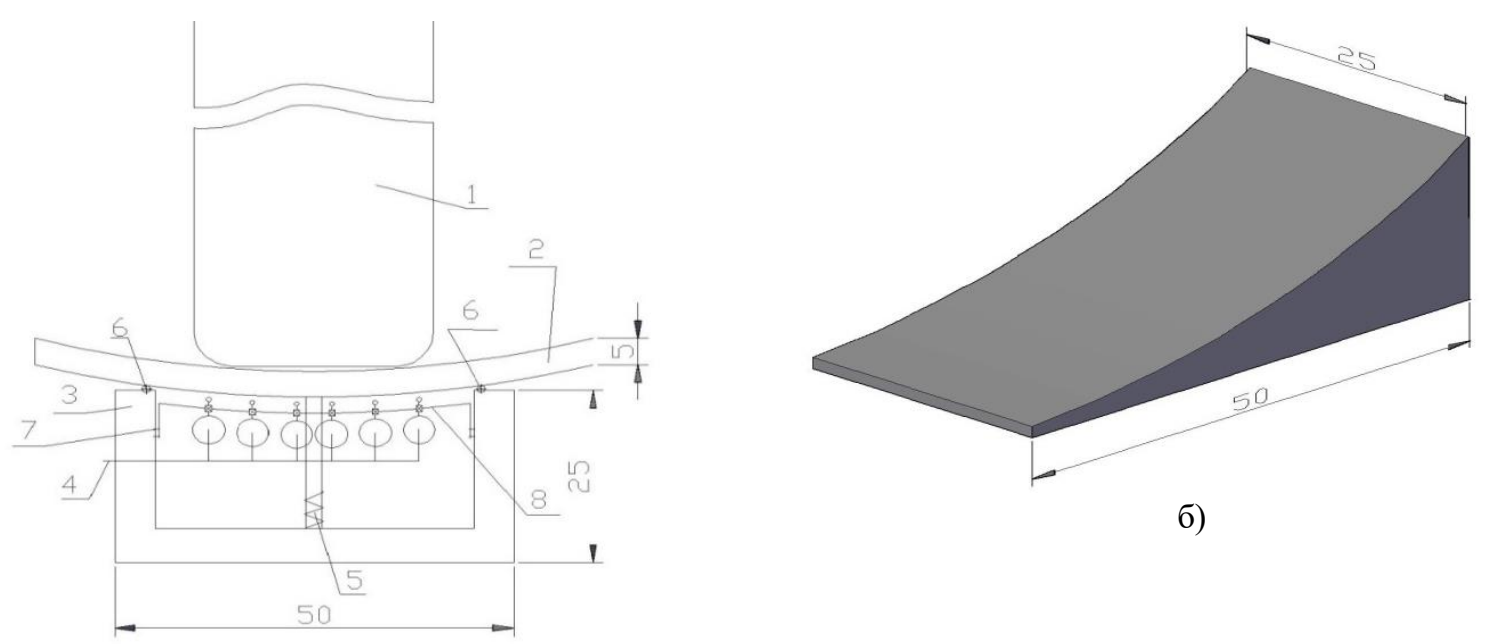

б)

a)

Рис.3. Приспособление для определения вертикальной нагрузки на почву: а-общая схема; б, в-трамплин для подъёма и опускания колеса трактора

1-шина (балон) ; 2-опора (тарелки); 3-рама; 4-индикаторы (7 шт); 5-регулировочные устройства (винт для подъёма и опускания тарелки); 6-опорные шарики (4 шт); 7-кронштейн для подъёма и опускания секции индикаторов; 8-регулировочные болты для индикаторов.

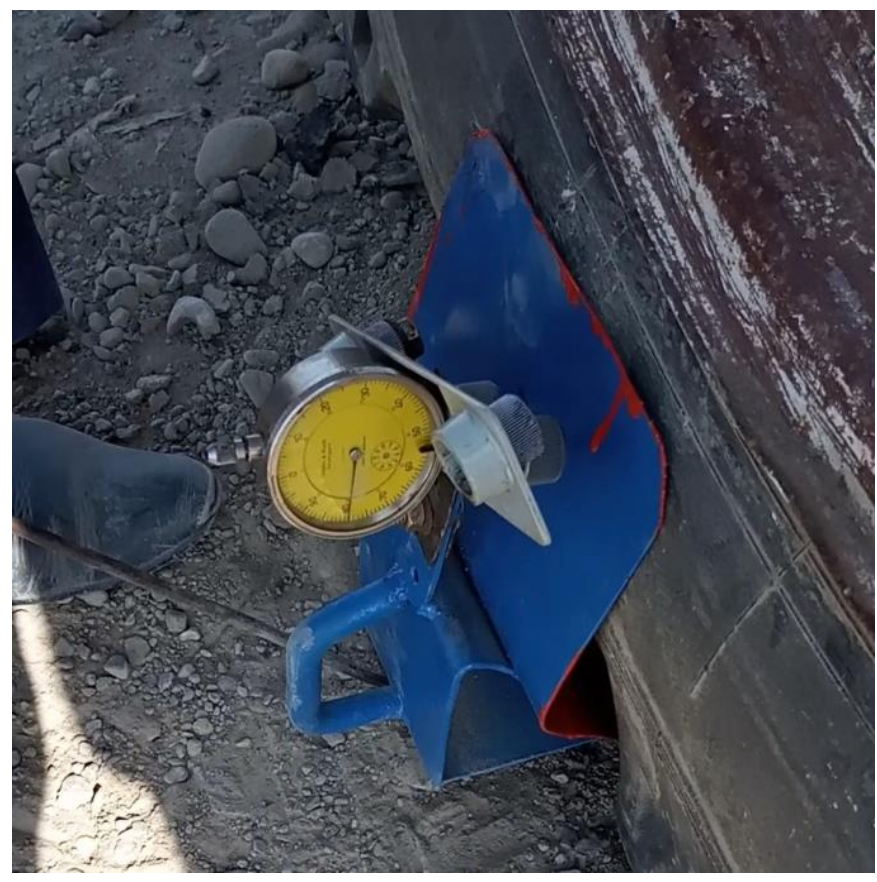

Рис.4. Ведущее колесо 13,6 R38 ЯP-318 для определения деформационных свойств при помощи индикатора часовой модели ИЧ-150 (давление в шинах 0,11 МПа) 


\begin{tabular}{|c|c|c|c|c|c|c|}
\hline \multirow{4}{*}{ Impact Factor: } & ISRA (India) & $=4.971$ & SIS (USA) & $=0.912$ & ICV (Poland) & $=6.630$ \\
\hline & ISI (Dubai, UAE & $=0.829$ & РИНЦ (Russia) & $=0.126$ & PIF (India) & $=1.940$ \\
\hline & GIF (Australia) & $=0.564$ & ESJI (KZ) & $=8.716$ & IBI (India) & $=4.260$ \\
\hline & JIF & $=1.500$ & SJIF (Morocco) & $=5.667$ & OAJI (USA) & $=0.350$ \\
\hline
\end{tabular}

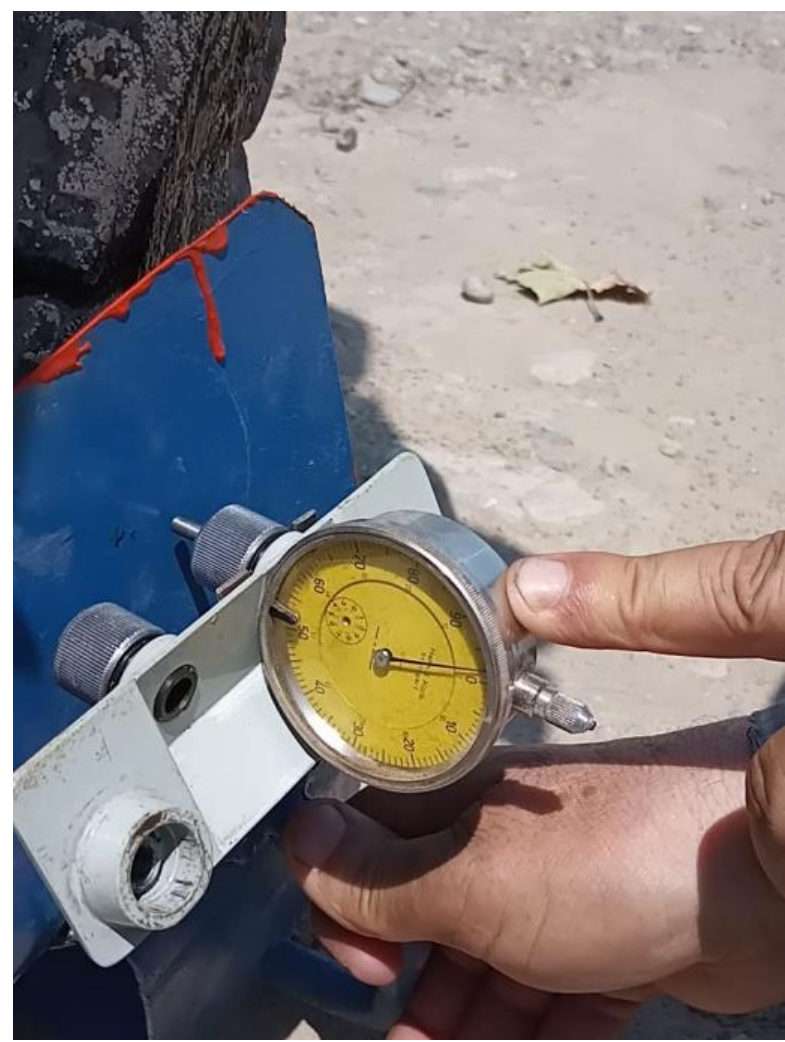

Рис.5. Установки для измерения деформации шин направлящего колеса 12-16 Л-163 при помощи индикатора часовой модели ИЧ-150 (давление в шинах 0,10 МПа)

Анализ приведённых зависимостей деформационных свойств тракторных шин показывает, что с увеличением нагрузки на почву, происходит уменьшение буксования и улучшение (Рис.3.4).

Деформационные свойства тракторных шин определяли на овальной поверхности (рис.5).

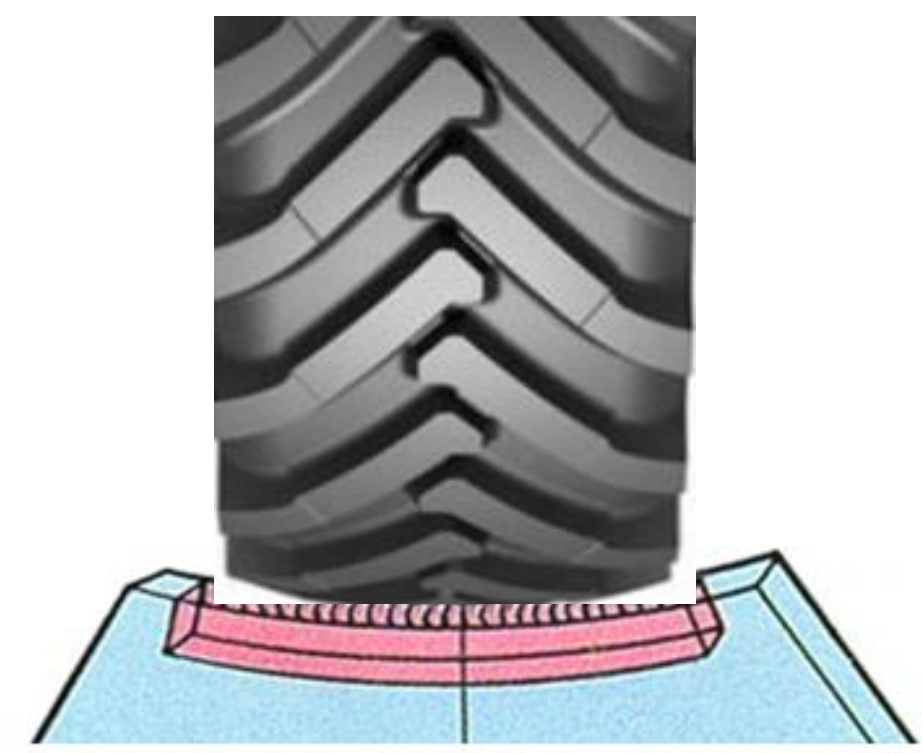

Рис.5. Площадь контакта шины с почвой в зависимости от нагрузки размеров шин: 1-балон; 2-площадь контакта. 


\begin{tabular}{|c|c|c|c|c|c|c|}
\hline \multirow{4}{*}{ Impact Factor: } & ISRA (India) & $=4.971$ & SIS (USA) & $=0.912$ & ICV (Poland) & $=6.630$ \\
\hline & ISI (Dubai, UAE) & $=0.829$ & РИНЦ (Russia) & $=0.126$ & PIF (India) & $=1.940$ \\
\hline & GIF (Australia) & $=0.564$ & ESJI (KZ) & $=8.716$ & IBI (India) & $=4.260$ \\
\hline & JIF & $=1.500$ & SJIF (Morocco) & $=5.667$ & OAJI (USA) & $=0.350$ \\
\hline
\end{tabular}

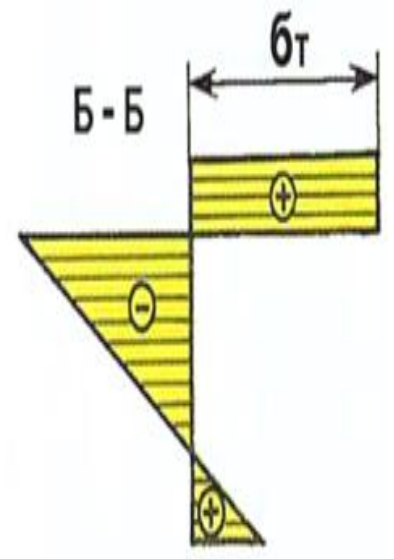

$\oplus$ - растяжение (нагрузка);

(-) - сжатие (разрузка).

$$
\sigma=\mathrm{Q} / \mathrm{F}_{\mathrm{k}}
$$

где: $\sigma$ - напряжение;

Q - сила (нагрузка) (500 кг).

$\mathrm{F}_{\mathrm{k}}=4 \mathrm{~h}_{\mathrm{z}}$

$\mathrm{F}_{\mathrm{k}}=$ площадь контакта; мм² $\mathrm{r}_{\mathrm{c}^{-}}$статический радиус; мм, В- ширина профиля; мм, $\mathrm{h}_{\mathrm{z}^{-}}$ нормальный прогиб, мм.

Таблица 1. Значения сечения площади контакта тракторных шин

\begin{tabular}{|c|c|c|c|}
\hline$\pi / \mathrm{H}$ & \multicolumn{2}{|c|}{ Нормальный прогиб, $\mathrm{h}_{\mathrm{z}} \mathrm{Mм}$} & Значение $\mathrm{F}_{\mathrm{k}}\left(\mathrm{мм}^{2}\right)$ \\
\hline 1 & $\mathrm{~h}_{1}$ & 0,61 & 37,74 \\
\hline 2 & $\mathrm{~h}_{2}$ & 0,42 & 26,38 \\
\hline 3 & $\mathrm{~h}_{3}$ & 0,57 & 23,23 \\
\hline 4 & $\mathrm{~h}_{4}$ & 0,45 & 28,88 \\
\hline 5 & $\mathrm{~h}_{5}$ & 0,68 & 42,70 \\
\hline
\end{tabular}

Построение эпюр изгибающих и крутящих моментов ведущего колеса универсального пропашного трактора «Hew Holland» [5,6].

$\mathrm{F}_{\mathrm{t} 1}=1100 \mathrm{H} ; \mathrm{F}_{\mathrm{r} 1}=1820 \mathrm{H} ; \mathrm{F}_{\mathrm{a} 1}=4900 \mathrm{H} ;$

$\mathrm{F}_{\mathrm{m}}=1100 H ; \mathrm{d}_{1}=80 \mu \mu ; \mathrm{L}_{5}=200 \mu \mu ;$

$\mathrm{L}_{\mathrm{m}}=44$ мм.

1). Вертикальная плоскость:

А). Определяем опорные реакции, $H$;

$\Sigma \mathrm{M}_{3}=\mathrm{Q} ;-\mathrm{R}_{\mathrm{ay}} \mathrm{L}_{\mathrm{B}}+\mathrm{Fa}_{1} \frac{\mathrm{d} 1}{2}-\mathrm{F}_{\mathrm{r} 1} \frac{\mathrm{Lb}}{2}=0 ;$

$\mathrm{R}_{\mathrm{ay}}=\frac{\mathrm{Fa} 1 \mathrm{~d} 1 / 2-\mathrm{Fr} 1 \mathrm{Lb} 1 / 2}{2}=$

$=4900 H \cdot 40 \cdot 10^{-3} \mathrm{M}-1820 H \cdot 100 \cdot 10^{-3} /(49 \cdot 40-1820)$ $H / 2=(20 \cdot 49-910) H=70 H$

$\Sigma \mathrm{M}_{1}=\mathrm{Q} ; \frac{\mathrm{Fr} 1 \mathrm{Lb}}{2}+\frac{\mathrm{Fa} 1 \mathrm{~d} 1}{2}-\mathrm{R}_{\mathrm{by}} \mathrm{L}_{\mathrm{b}}=0 ;$

$\mathrm{R}_{\mathrm{by}}=\frac{\operatorname{Fr} 1 \mathrm{Lb} / 2+F a \mathrm{~d} 1 / 2}{2}=$

$=1820 \mathrm{H} \cdot 100 \cdot 10^{-3} \mathrm{M+}$

$+4900 H \cdot 40 \cdot 10^{-3}{ }^{M} / 5-200 \cdot 10^{-3} \mathcal{M}=1890 \mathrm{H}$,

Проверка: $\Sigma \mathrm{V}=\mathrm{Q} ; \mathrm{R}_{\mathrm{by}}-\mathrm{F}_{\mathrm{r} 1}-\mathrm{R}_{\mathrm{ay}}=0$;

Б). Построение эпюры изгибающих моментов относительно оси $X$ в характерных сечениях 1,3 Нм:

$\Sigma \mathrm{M}_{\mathrm{x} 1}=\mathrm{Q} ; \mathrm{M}_{\mathrm{x} 2}=-\mathrm{R}_{\mathrm{ay}} \mathrm{L}_{\mathrm{b}} / 2 ; \mathrm{M}_{\mathrm{x} 3}=0 ;$
$\mathrm{M}_{\mathrm{x} 2}=\mathrm{R}_{\mathrm{ay}} \mathrm{L}_{\mathrm{b}} / 2$.

2. Горизонтальная плоскость

А) определяем опорные реакции, $H$.

$$
\begin{aligned}
& \Sigma \mathrm{M}_{3}=\mathrm{Q} ; \quad \mathrm{F}_{\mathrm{M}} \mathrm{L}_{\mathrm{M}}+\frac{\mathrm{Ft} 1 \mathrm{Lb}}{2}-\mathrm{R}_{\mathrm{ax}} \mathrm{L}_{\mathrm{b}}=0 ; \\
& \mathrm{R}_{\mathrm{ax}}=\mathrm{F}_{\mathrm{M}} \mathrm{L}_{\mathrm{M}}+\frac{\mathrm{FmLm}+\mathrm{Ft} 1 \mathrm{Lb}}{2}=53,1 \mathrm{H}
\end{aligned}
$$

$\cdot 4,4 \cdot 10^{-3} \mathcal{M}+1100 H \cdot 100 \cdot 10^{-3} \mathcal{M} / 200 \cdot 10^{-3} \mathcal{M}=666,82$ $H$;

$\Sigma \mathrm{M}_{1}=\mathrm{Q} ;-\mathrm{R}_{\mathrm{bx}} \mathrm{L}_{\mathrm{b}}-\mathrm{Ft}_{1} \mathrm{~L}_{\mathrm{b}} / 2+\mathrm{F}_{\mathrm{M}}\left(\mathrm{L}_{\mathrm{M}}+\mathrm{L}_{\mathrm{b}}\right)=0$;

$\mathrm{R}_{\mathrm{bx}}=-\mathrm{Ft}_{1} \mathrm{~L}_{\mathrm{b}} / 2+\mathrm{F}_{\mathrm{M}}\left(\mathrm{L}_{\mathrm{M}}+\mathrm{L}_{\mathrm{b}}\right) / \mathrm{L}_{\mathrm{b}}=-1100 H \cdot 100$ $\cdot 10^{-3} \mathcal{M}+531 \mathrm{H} \cdot 244 \cdot 10^{-3} \mathrm{M} / 200 \cdot 10^{-3} \mathrm{M}=-110000 \mathrm{H}$ $+12956 H / 200=97,82 H$

Б). Построение эпюры изгибающих моментов относительно оси $У$ в характерных сечениях $1 \ldots 4, \mathrm{H} / \mathrm{s}$;

$\Sigma \mathrm{M}_{\mathrm{y} 1}=\mathrm{Q} ; \mathrm{M}_{\mathrm{y} 2}=-\frac{\mathrm{Ra} \mathrm{Lb}}{2} ; \mathrm{M}_{\mathrm{y} 4}=0$;

$\mathrm{M}_{\mathrm{y} 3}=-\mathrm{F}_{\mathrm{m}} \mathrm{L}_{\mathrm{on}}$. H.м

3. Построение эпюры крутящих моментов,

$\mathrm{M}_{\mathrm{n}}=\mathrm{M}_{\mathrm{z}}=\frac{\mathrm{Ft} 1 \mathrm{~d} 1}{2}=1100 H \cdot 40 \cdot 10^{-3} \mathrm{M} / 2=$

$=22 \mathrm{H} \cdot \mathrm{m}$; 


\begin{tabular}{|c|c|c|c|c|c|c|}
\hline \multirow{4}{*}{ Impact Factor: } & ISRA (India) & $=4.971$ & SIS (USA) & $=0.912$ & ICV (Poland) & $=6.630$ \\
\hline & ISI (Dubai, UAE & $=0.829$ & РИНЦ (Russia) & $=0.126$ & PIF (India) & $=1.940$ \\
\hline & GIF (Australia) & $=0.564$ & ESJI (KZ) & $=8.716$ & IBI (India) & $=4.260$ \\
\hline & JIF & $=1.500$ & SJIF (Morocco) & $=5.667$ & OAJI (USA) & $=0.350$ \\
\hline
\end{tabular}

4. Определяем суммарные радиальные реакции, $\mathrm{H}$

$$
\begin{aligned}
& \mathrm{R}_{\mathrm{a}}=\sqrt{\operatorname{Rax}^{2}-R a y^{2}}=670,484 H ; \\
& \mathrm{R}_{\mathrm{a}}=\sqrt{R b x^{2}-R b y^{2}}=1892,52 H .
\end{aligned}
$$

5. Определяем суммарные изгибающие моменты в наиболее нагруженных сечениях, $H \cdot m$; $\mathrm{M}_{\mathrm{z}}=\sqrt{M x^{2}-M y^{2}}=1821 H . \mathrm{M}_{3}=\mathrm{M}_{\mathrm{y} 3}$

\begin{tabular}{|c|c|}
\hline $\mathrm{R}_{\mathrm{A}, \mathrm{H}}$ & 670 \\
\hline $\mathrm{R}_{\mathbf{B}, \mathrm{H}}$ & 1893 \\
\hline $\mathrm{M}_{2}, \mathrm{HM}$ & 202,7 \\
\hline $\mathrm{M}_{3}, \mathrm{HM}$ & 23,36 \\
\hline
\end{tabular}

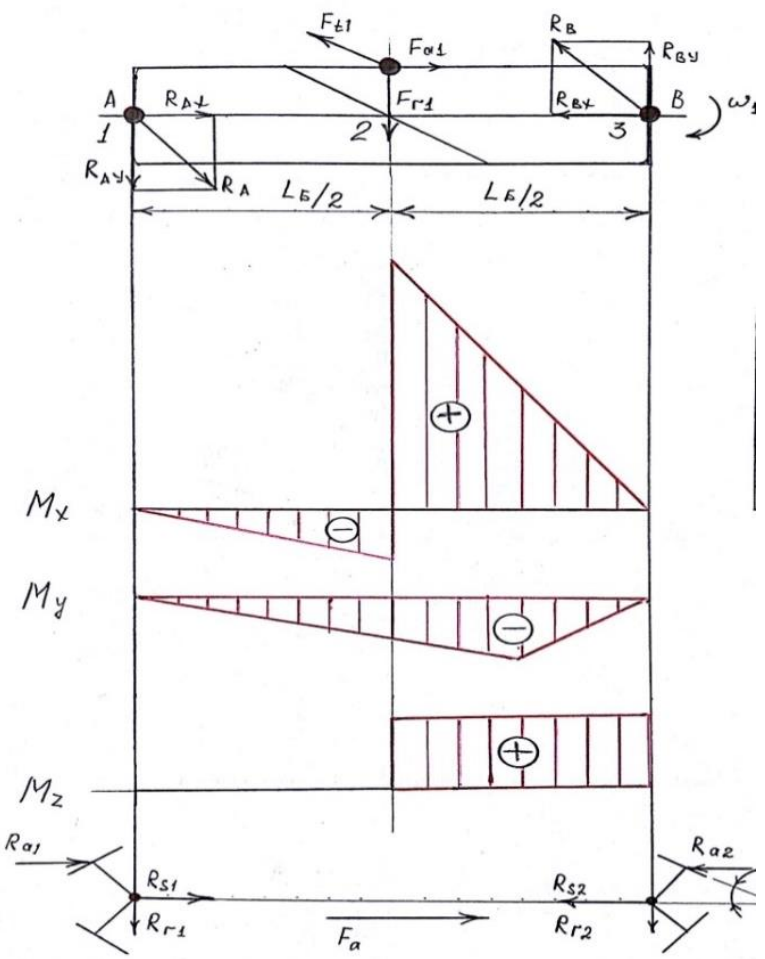

Рис.6. Расчётные схемы ведущего колеса трактора «Нью Холланд»

Значения изгибающих, крутящих моментов и суммарных радиальных реакций получены по исходным данным.

Деформация шины при контакте с опорной поверхностью борозды ведущего колеса трактора оказывает, значительное влияние на тягово- сцепные качества и проходимость трактора. Площадь контакта тракторных пневматическых шин, непосредственно связанную с нормальной деформацией, определена экспериментально на твёрдом основании при нормальном воздухе шины.

\section{References:}

1. Kostenko, M.Ju., Beznostjuk, R.V., \& Nushtaev, N.N. (2018). Povyshenie jeffektivnosti tehnicheskogo obsluzhivanija, remonta $i$ diagnostirovanija sel'skohozjajstvennoj tehniki. «Innovacionnoe nauchno-obrazovatel'noe obespechenie agropromyshlennogo kompleksa» materialy 69-oj Mezhdunarodnoj nauch.-prakt. konf. (chast' II). (pp.161-163). Rjazan': RGATU.
2. Melibaev, M., Dadakhodjaev, A., \& Mamadjonov, M. (2019). Features of the naturalindustral conditions of the zone and operation of machine-tractor units. ACADEMICIA An International Multidisciplinary Research Jonrnal. ISSN 2249-7137. Vol 9 Issue 3, March 2019. Impact Factor SJIF 2018=6.152. India. 2019. -p. 37-41. (10.5958/22497137.2019.00033.8). 


\begin{tabular}{|c|c|c|c|c|c|c|}
\hline \multirow{4}{*}{ Impact Factor: } & ISRA (India) & $=4.971$ & SIS (USA) & $=0.912$ & ICV (Poland) & $=6.630$ \\
\hline & ISI (Dubai, UAE & $=0.829$ & РИНЦ (Russia) & $=0.126$ & PIF (India) & $=1.940$ \\
\hline & GIF (Australia) & $=0.564$ & ESJI (KZ) & $=8.716$ & IBI (India) & $=4.260$ \\
\hline & JIF & $=1.500$ & SJIF (Morocco) & $=5.667$ & OAJI (USA) & $=0.350$ \\
\hline
\end{tabular}

3. Melibayev, M. (2019). Capacity of universalwell-towed-wheel tires. Scientific-technical journal of FerPi. ISSN 2181-7200. Vol.4. 2019. Fergana, pp. 53-61.

4. Naderi-Boldaji, M., et al. (2013). 3D finite element simulation of a single-tip horizontal penetrometer-soil interaction. Part I: development of the model and evaluation of the model parameters. Soil Tillage Res. 134, pp.153162.
5. Jy̆ldashev, Sh.U. (1994). Mashinalar ishonchliligi va ularni ta\#mirlash asoslari. (p.479). Toshkent: "Y̌zzekiston".

6. (n.d.). Retrieved from http://www.dissercat.com/content/povyshenieeffektivnosti-ekspluatatsii-traktorov-putemobespecheniya-ikh-rabotosposobnostid\#ixzz5STor5r4y 